\title{
CHARACTERIZATION OF CURVED CREASES AND RULINGS: DESIGN AND ANALYSIS OF LENS TESSELLATIONS
}

\author{
ERIK D. DEMAINE, MARTIN L. DEMAINE, DAVID A. HUFFMAN, DUKS KOSCHITZ, \\ AND TOMOHIRO TACHI
}

\begin{abstract}
We describe a general family of curved-crease folding tessellations consisting of a repeating "lens" motif formed by two convex curved arcs. The third author invented the first such design in 1992, when he made both a sketch of the crease pattern and a vinyl model (pictured below). Curve fitting suggests that this initial design used circular arcs. We show that in fact the curve can be chosen to be any smooth convex curve without inflection point. We identify the ruling configuration through qualitative properties that a curved folding satisfies, and prove that the folded form exists with no additional creases, through the use of differential geometry.
\end{abstract}

\section{INTRODUCTION}

The past two decades have seen incredible advances in applying mathematics and computation to the analysis and design of origami made by straight creases. But we lack many similar theorems and algorithms for origami made by curved creases.

In this paper, we develop several basic tools (definitions and theorems) for curved-crease origami. These tools in particular characterize the relationship between the crease pattern and rule lines/segments, and relate creases connected by rule segments. Some of these tools have been developed before in other contexts (e.g., Fuchs and Tabachnikov 99, Fuchs and Tabachnikov 07, Huffman 76), but have previously lacked a careful analysis of the levels of smoothness $\left(C^{1}, C^{2}\right.$, etc.) and other assumptions required. Specific high-level properties we prove include:

(1) Regions between creases decompose into noncrossing rule segments, which connect from curved crease to curved crease, and planar patches (a result from Demaine et al. 11).

(2) The osculating plane of a crease bisects the two adjacent surface tangent planes (when they are unique).

(3) A curved crease with an incident cone ruling (a continuum of rule segments at a point) cannot fold smoothly: it must be kinked at the cone ruling.

(4) Rule segments on the convex side of a crease bend mountain/valley the same as the crease, and rule segments on the concave side of a crease bend mountain/valley opposite from the crease.

E. Demaine and M. Demaine supported in part by NSF ODISSEI grant EFRI-1240383 and NSF Expedition grant CCF-1138967.

D. Koschitz performed this research while at MIT.

T. Tachi supported by the JST Presto program. 
(5) If two creases are joined by a rule segment on their concave sides, or on their convex sides, then their mountain/valley assignments must be equal. If the rule segment is on the convex side of one crease and the concave side of the other crease, then the mountain/valley assignments must be opposite.

We apply these tools to analyze one family of designs called the lens tessellation. Figure 1 shows an example originally designed and folded by the third author in 1992, and now modeled digitally. We prove that this curved crease pattern folds into 3D, with the indicated rule segments, when the "lens" is any smooth convex curve. We also show that the model is "rigidly foldable", meaning that it can be continuously folded without changing the ruling pattern.

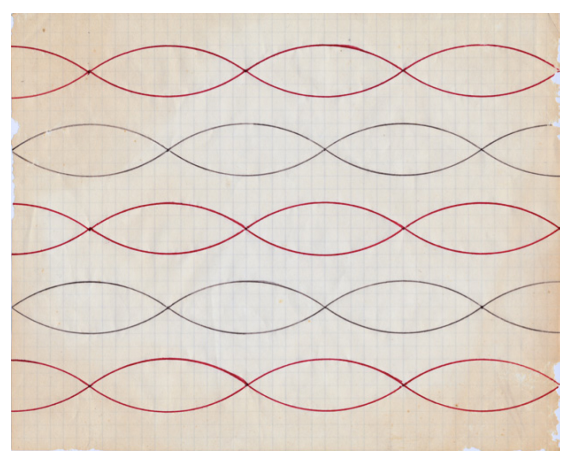

(A) Huffman's original hand-drawn sketch of crease pattern of lens design (1992).

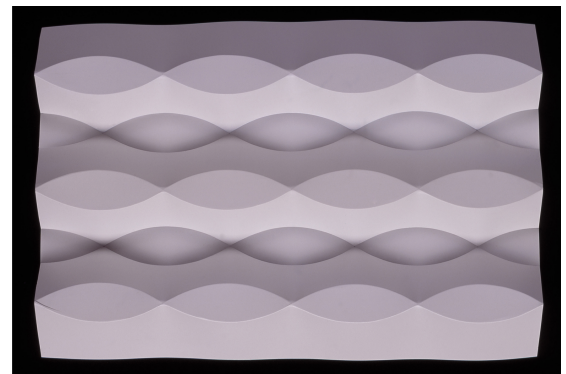

(c) Huffman's original hand-folded vinyl model (1992). Photo by Tony Grant.

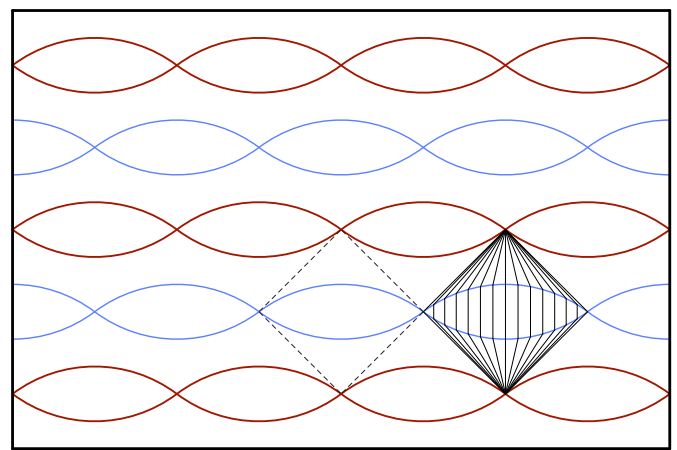

(B) Computer-drawn crease pattern of lens design.

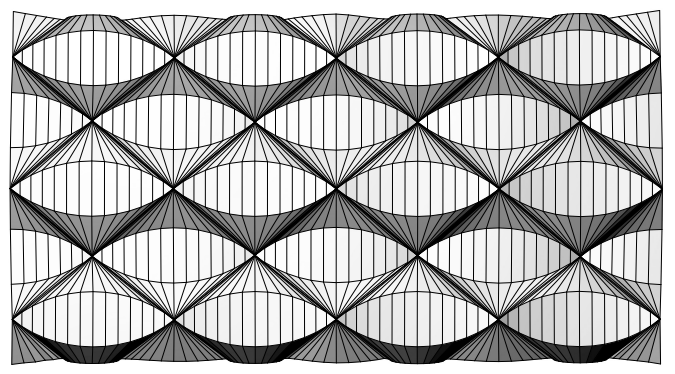

(D) Computer-simulated 3D model using Tachi's Freeform Origami software.

Figure 1. Lens tessellation: 1992 original (left) and digital reconstruction (right).

The 3D configuration of the curved folding is solved through identifying the correspondence between pairs of points connected by rule segments, using the qualitative properties described above. These properties separate the tessellation into independent kite-shaped tiles and force the rulings between the lenses to be particular cones with their apices coinciding with the vertices of the tiling. The ruling inside each 
lens is free (can twist), but assuming no twist or global planarity/symmetry, is cylindrical (vertical rule segments). The tiling exists by rotation/reflection of the 3D model of each kite around its four straight boundary edges. From the tiling symmetry, each tile edge has a common tangent to its neighbors regardless of the type of curves, as long as it is a convex curve.

The rest of this paper is organized as follows. Section 2 introduces some basic notation for 2D and 3D curves. Section 3 defines creases, crease patterns, foldings, rule segments, cone ruling, orientation of the paper, and surface normals (and analyzes when they exist). Section 4 proves that the powerful bisection propertythe osculating plane of a crease bisects the two adjacent surface tangent planes - and uses it to rule out some strange situations such as rule segments tangent to creases or zero-length rule segments. Section 5 characterizes smooth folding: a crease is folded $C^{1}$ if and only if it is folded $C^{2}$ if and only if there are no incident cone rulings. Section 6 defines mountains and valleys for both creases and the bending of rule segments, and relates the two. Finally, Section 7 uses all these tools to analyze lens tessellations, proving a necessary and sufficient condition on their foldability.

\section{Curves}

In this section, we define some standard parameterizations of curves in $2 \mathrm{D}$ and $3 \mathrm{D}$, which we will use in particular for describing creases in the unfolded paper and folded state. Our notation introduces a helpful symmetry between 2D (unfolding) and 3D (folding): lower case indicates 2D, while upper case indicates the corresponding notion in 3D.

2.1. 2D Curves. Consider an arclength-parameterized $C^{2} 2 \mathrm{D}$ curve $\mathbf{x}:(0, \ell) \rightarrow$ $\mathbb{R}^{2}$ (or in any metric 2-manifold). For $s \in(0, \ell)$, define the (unit) tangent at $s$ by

$$
\mathbf{t}(s)=\frac{d \mathbf{x}(s)}{d s} \text {. }
$$

Define the curvature

$$
k(s)=\left\|\frac{d \mathbf{t}(s)}{d s}\right\| .
$$

In particular, call the curve curved at $s$ if its curvature $k(s)$ is nonzero. In this case, define the (unit) normal at $s$ by

$$
\mathbf{n}(s)=\frac{d \mathbf{t}(s)}{d s} / k(s) .
$$

The curve is curved (without qualification) if it is curved at all $s \in(0, \ell)$.

Define the convex side at $s$ to consist of directions having negative dot product with $\mathbf{n}(s)$; and define the concave side at $s$ to consist of directions having positive dot product with $\mathbf{n}(s)$.

2.2. 3D Curves. For an arclength-parameterized $C^{2}$ space curve $\mathbf{X}:[0, \ell] \rightarrow \mathbb{R}^{3}$, and for a parameter $s \in[0, \ell]$ inducing a point $\mathbf{X}(s)$, define the (unit) tangent

$$
\mathbf{T}(s)=\frac{d \mathbf{X}(s)}{d s} .
$$

Define the curvature

$$
K(s)=\left\|\frac{d \mathbf{T}(s)}{d s}\right\|
$$


In particular, call the curve curved at $s$ if its curvature $K(s)$ is nonzero (and curved without qualification if it is curved at all $s \in(0, \ell)$ ). In this case, define the (unit) normal at $s$ by

$$
\mathbf{N}(s)=\frac{d \mathbf{T}(s)}{d s} / K(s)
$$

define the (unit) binormal

$$
\mathbf{B}(s)=\mathbf{T}(s) \times \mathbf{N}(s)
$$

and define the torsion

$$
\tau(s)=-\frac{d \mathbf{B}(s)}{d s} \cdot \mathbf{N}(s) .
$$

Equivalently, these definitions follow from the Frenet-Serret formulas:

$$
\left[\begin{array}{ccc}
0 & K(s) & 0 \\
-K(s) & 0 & \tau(s) \\
0 & -\tau(s) & 0
\end{array}\right] \cdot\left[\begin{array}{l}
\mathbf{T}(s) \\
\mathbf{N}(s) \\
\mathbf{B}(s)
\end{array}\right]=\frac{d}{d s}\left[\begin{array}{l}
\mathbf{T}(s) \\
\mathbf{N}(s) \\
\mathbf{B}(s)
\end{array}\right]
$$

Lemma 1. For any curved $C^{2} 3 D$ curve $\mathbf{X}(s)$, the Frenet frame $(\mathbf{T}(s), \mathbf{N}(s), \mathbf{B}(s))$ and curvature $K(s)$ exist and are continuous.

Proof. Because $\mathbf{X}(s)$ is differentiable, $\mathbf{T}(s)$ exists. Because $\mathbf{X}(s)$ is twice differentiable, $K(s)$ exists, and because $\mathbf{X}(s)$ is $C^{2}, K(s)$ is continuous. Because the curve is curved, $K(s) \neq 0$, so we do not divide by 0 in computing $\mathbf{N}(s)$, and thus $\mathbf{N}(s)$ exists and is continuous. The cross product in $\mathbf{B}(s)$ exists and is continuous because $\mathbf{T}(s)$ and $\mathbf{N}(s)$ are guaranteed to be normalized (hence nonzero) and orthogonal to each other (hence not parallel).

The same lemma specializes to $2 \mathrm{D}$, by dropping the $\mathbf{B}(s)$ part:

Corollary 2. For any curved $C^{2} 2 D$ curve $\mathbf{x}(s)$, the frame $(\mathbf{t}(s), \mathbf{n}(s))$ curvature $k(s)$ exist and are continuous.

\section{Foldings}

The following definitions draw from [Demaine et al. 11, Demaine and O'Rourke 07].

We start with 2D (unfolded) notions. A piece of paper is an open 2-manifold embedded in $\mathbb{R}^{2}$. A crease $\mathbf{x}$ is a $C^{2} 2 \mathrm{D}$ curve contained in the piece of paper and not self-intersecting (i.e., not visiting the same point twice). A crease point is a point $\mathbf{x}(s)$ on the relative interior of the crease (excluding endpoints). The endpoints of a crease are vertices. A crease pattern is a collection of creases that meet only at common vertices. Equivalently, a crease pattern is an embedded planar graph, where each edge is embedded as a crease. This definition effectively allows piecewise- $C^{2}$ curves, by subdividing the edge in the graph with additional vertices; "creases" are the resulting $C^{2}$ pieces. A face is a maximal open region of the piece of paper not intersecting any creases or vertices.

Now we proceed to 3D (folded) notions. A (proper) folding of a crease pattern is a piecewise- $C^{2}$ isometric embedding of the piece of paper into $3 \mathrm{D}$ that is $C^{1}$ on every face and not $C^{1}$ at every crease point and vertex. Here isometric means that intrinsic path lengths are preserved by the mapping; and piecewise- $C^{2}$ means that the folded image can be decomposed into a finite complex of $C^{2}$ open regions joined by points and $C^{2}$ curves. We use the terms folded crease, folded vertex, folded face, and folded piece of paper to refer to the image of a crease, vertex, face, and entire piece of paper under the folding map. Thus, each folded face subdivides into a 
finite complex of $C^{2}$ open regions joined by points called folded semivertices and $C^{2}$ curves called folded semicreases. Each folded crease $\mathbf{X}(s)$ can be subdivided into a finite sequence of $C^{2}$ curves joined by $C^{1}$ points called semikinks and not- $C^{1}$ points called kinks. (Here $C^{1} /$ not- $C^{1}$ is a property measured of the crease $\mathbf{X}(s)$; crease points are necessarily not $C^{1}$ on the folded piece of paper.) In fact, semivertices do not exist [Demaine et al. 11, Corollary 2], and neither do semikinks (Corollary 20 below).

Lemma 3. A curved crease $\mathbf{x}(s)$ folds into a $3 D$ curve $\mathbf{X}(s)$ that contains no line segments (and thus is curved except at kinks and semikinks).

Proof. Suppose $X(s)$ is a $3 \mathrm{D}$ line segment for $s \in\left[s_{1}, s_{2}\right]$. Then the distance between $\mathbf{X}\left(s_{1}\right)$ and $\mathbf{X}\left(s_{2}\right)$ as measured on the folded piece of paper is the length of this line segment, i.e., the arc length of $\mathbf{X}$ over $s \in\left[s_{1}, s_{2}\right]$ which, by isometry, equals the arc length of $\mathbf{x}$ over $s \in\left[s_{1}, s_{2}\right]$. However, in the 2D piece of paper, there is a shorter path connecting $\mathbf{x}\left(s_{1}\right)$ and $\mathbf{x}\left(s_{2}\right)$ because the $2 \mathrm{D}$ crease is curved (and not on the paper boundary, because the paper is an open set), contradicting isometry.

3.1. Developable Surfaces. A folded face is also known as an uncreased developable surface: it is uncreased in the sense that it is $C^{1}$, and developable in the sense that every point $p$ has a neighborhood isometric to a region in the plane. The following theorem from Demaine et al. 11 characterizes what uncreased developable surfaces look like:

Theorem 4 (Corollaries 1-3 of [Demaine et al. 11]). Every interior point $p$ of an uncreased developable surface $M$ not belonging to a planar neighborhood belongs to a unique rule segment $C_{p}$. The rule segment's endpoints are on the boundary of $M$. In particular, every semicrease is such a rule segment.

Corollary 5. Any folded face decomposes into planar regions and nonintersecting rule segments (including semicreases) whose endpoints lie on creases.

For a folded piece of paper, we use the term (3D) rule segment for exactly these segments $C_{p}$ computed for each folded face, for all points $p$ that are not folded vertices, not folded crease points, and not belonging to a planar neighborhood. In particular, we view the interior of planar regions as not containing any rule segments (as they would be ambiguous); however, the boundaries of planar regions are considered rule segments. As a consequence, all rule segments have a neighborhood that is nonplanar.

For each 3D rule segment in the folded piece of paper, we can define the corresponding $2 \mathrm{D}$ rule segment by the inverse mapping. By isometry, $2 \mathrm{D}$ rule segments are indeed line segments.

Define a cone ruling at a crease point $\mathbf{x}(s)$ to be a fan of $2 \mathrm{D}$ rule segments emanating from $\mathbf{x}(s)$ in a positive-length interval of directions $\left[\theta_{1}, \theta_{2}\right]$.

3.2. Orientation. We orient the piece of paper in the $x y$ plane by a consistent normal $\mathbf{e}_{z}$ (in the $+z$ direction) called the top side. This orientation defines, for a $2 \mathrm{D}$ crease $\mathbf{x}=\mathbf{x}(s)$ in the crease pattern, a left normal $\hat{\mathbf{n}}(s)=\mathbf{e}_{z} \times \mathbf{t}(s)$. Where $\mathbf{x}(s)$ is curved and thus $\mathbf{n}(s)$ is defined, we have $\hat{\mathbf{n}}(s)= \pm \mathbf{n}(s)$ where the sign specifies whether the left or right side corresponds to the convex side of the curve. We can also characterize a $2 \mathrm{D}$ rule segment incident to $\mathbf{x}(s)$ as being left of $\mathbf{x}$ when 
the vector emanating from $\mathbf{x}(s)$ has positive dot product with $\hat{\mathbf{n}}(s)$, and right of $\mathbf{x}$ when it has negative dot product. (In Lemma 12 below, we prove that no rule segment is tangent to a crease, and thus every rule segment is either left or right of the crease.)

We can also define the signed curvature $\hat{k}(s)$ to flip sign where $\hat{\mathbf{n}}(s)$ does: $\hat{k}(s) \hat{\mathbf{n}}(s)=k(s) \mathbf{n}(s)$. Then $\hat{k}(s)$ is positive where the curve turns left and negative where the curve turns right (relative to the top side).

3.3. Unique Ruling. Call a crease point $\mathbf{x}(s)$ uniquely ruled on the left if there is exactly one rule segment left of $\mathbf{x}(s)$; symmetrically define uniquely ruled on the right; and define uniquely ruled to mean uniquely ruled on both left and right.

By Corollary 5 , there are two possible causes for a crease point $\mathbf{x}(s)$ to be not uniquely ruled (say on the left). First, there could be one or more cone rulings (on the left) at $\mathbf{x}(s)$. Second, there could be one or more planar 3D regions incident to $\mathbf{X}(s)$ (which, in $2 \mathrm{D}$, lie on the left of $\mathbf{x}(s)$, meaning the points have positive dot product with $\hat{\mathbf{n}}(s))$.
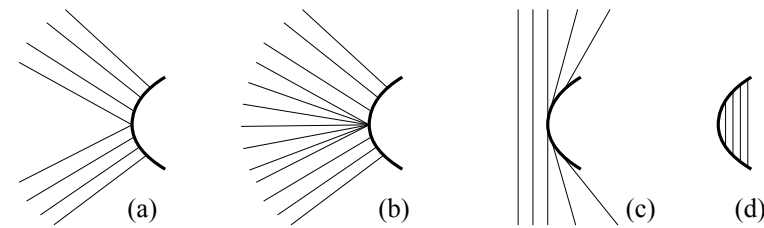

Figure 2. Possibilities for a crease to be not uniquely ruled.

One special case of unique ruling is when a rule segment is tangent to a curved crease. Ultimately, in Lemma 12, we will prove that this cannot happen, but for now we need that the surface normals remain well-defined in this case. There are two subcases depending on whether the rule segment is on the convex or concave side of the crease, as in Figures 2(c) and (d). The rule segment's direction in 3D and surface normal vector remain well-defined in this case, by taking limits of nearby rule segments. In the concave subcase (d), we take the limit of rule segments on the same side of the curve. In the convex subcase (c), the rule segment splits the surface locally into two halves, and we take the limit of rule segments in the half not containing the crease. Because the surface normals are thus well-defined, we do not need to distinguish this case in our proofs below.

Call a crease point $\mathbf{x}(s)$ cone free if there are no cone rulings at $\mathbf{x}(s)$; similarly define cone free on the left/right. Such a point may still have a planar region, but only one:

Lemma 6. If a crease point $\mathbf{x}(s)$ is cone free, then it has at most one planar region on each side.

Proof. Refer to Figure 3 . Suppose $\mathbf{x}(s)$ had at least two planar regions on, say, the left side. Order the regions clockwise around $\mathbf{x}(s)$, and pick two adjacent planar regions $R_{1}$ and $R_{2}$. By Corollary 5 , the wedge with apex $\mathbf{x}(s)$ between $R_{1}$ and $R_{2}$ must be covered by rule segments. But by Theorem 4 , a rule segment cannot have its endpoints on the boundaries of $R_{1}$ and $R_{2}$, as it must extend all the way to creases. Thus the only way to cover the wedge locally near $\mathbf{x}(s)$ is to have a cone ruling at $\mathbf{x}(s)$. 


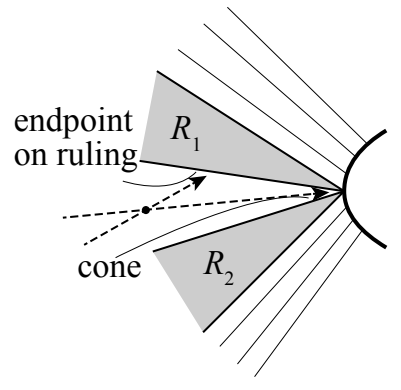

Figure 3. Two adjacent planar regions at a point.

3.4. Surface Normals. In $3 \mathrm{D}$, the orientation defines a top-side normal vector at every $C^{1}$ point $!^{1}$ For a crease point $\mathbf{X}(s)$ that is cone free on the left, we can define a unique left surface normal $\mathbf{P}_{L}(s)$. First, if there is a planar region on the left of $\mathbf{X}(s)$, then by Lemma 6 there is only one such planar region, and we define $\mathbf{P}_{L}(s)$ to be the unique top-side normal vector of the planar region. Otherwise, $\mathbf{X}(s)$ is uniquely ruled on the left, and we define $P_{L}(s)$ to be the top-side surface normal vector which is constant along this unique rule segment. (As argued above, this definition makes sense even when the rule segment is a zero-length limit of rule segments.) Similarly, we can define the right surface normal $\mathbf{P}_{R}(s)$ when $\mathbf{X}(s)$ is cone free on the right.

\section{Bisection Property}

In this section, we prove that, at a cone-free folded curved crease, the binormal vector bisects the left and right surface normal vectors, which implies that the osculating plane of the crease bisects the two surface tangent planes. Proving this bisection property requires several steps along the way, and has several useful consequences.

4.1. $C^{\mathbf{2}}$ Case. First we prove the bisection property at $C^{2}$ crease points, using the following simple lemma:

Lemma 7. For a $C^{2}$ folded curved crease $\mathbf{X}(s)$ that is cone-free on the left,

$$
(K(s) \mathbf{N}(s)) \cdot\left(\mathbf{P}_{L}(s) \times \mathbf{T}(s)\right)=\hat{\mathbf{k}}(s) .
$$

For a $C^{2}$ folded curved crease $\mathbf{X}(s)$ that is cone-free on the right,

$$
(K(s) \mathbf{N}(s)) \cdot\left(\mathbf{P}_{R}(s) \times \mathbf{T}(s)\right)=\hat{\mathbf{k}}(s) .
$$

Proof. We prove the left case; the right case is symmetric. The left-hand side is known as the geodesic curvature at $\mathbf{X}(s)$ on surface $S_{L}$, and is known to be invariant under isometry. In the unfolded $2 \mathrm{D}$ state, the geodesic curvature is

$$
(k(s) \mathbf{n}(s)) \cdot\left(\mathbf{e}_{z} \times \mathbf{t}(s)\right)=(k(s) \mathbf{n}(s)) \cdot \hat{\mathbf{n}}(s)=\hat{k}(s) .
$$

\footnotetext{
${ }^{1}$ For example, take infinitesimally small triangles around the point, oriented counterclockwise in $2 \mathrm{D}$, and compute their normals in $3 \mathrm{D}$.
} 
Lemma 8. For a $C^{2}$ cone-free folded curved crease $\mathbf{X}(s), \mathbf{B}(s)$ bisects $\mathbf{P}_{L}(s)$ and $\mathbf{P}_{R}(s)$. In particular, the tangent planes of the surfaces on both sides of $\mathbf{X}(s)$ form the same angle with the osculating plane.

Proof. A $C^{2}$ cone-free folded curved crease $\mathbf{X}(s)$ has unique left and right surface normals $\mathbf{P}_{L}(s)$ and $\mathbf{P}_{R}(s)$. By Lemma 7, the left and right geodesic curvatures match:

$$
(K(s) \mathbf{N}(s)) \cdot\left(\mathbf{P}_{L}(s) \times \mathbf{T}(s)\right)=(K(s) \mathbf{N}(s)) \cdot\left(\mathbf{P}_{R}(s) \times \mathbf{T}(s)\right) .
$$

The $K(s)$ scalars cancel, leaving a triple product:

$$
\mathbf{N}(s) \cdot\left(\mathbf{P}_{L}(s) \times \mathbf{T}(s)\right)=\mathbf{N}(s) \cdot\left(\mathbf{P}_{R}(s) \times \mathbf{T}(s)\right),
$$

which is equivalent to

$$
\mathbf{P}_{L}(s) \cdot(\mathbf{T}(s) \times \mathbf{N}(s))=\mathbf{P}_{R}(s) \cdot(\mathbf{T}(s) \times \mathbf{N}(s)) .
$$

Therefore $\mathbf{B}(s)=\mathbf{T}(s) \times \mathbf{N}(s)$ forms the same angle with $\mathbf{P}_{L}(s)$ and $\mathbf{P}_{R}$. Because $\mathbf{B}, \mathbf{P}_{L}$ and $\mathbf{P}_{R}$ lie in a common plane orthogonal to $\mathbf{T}, \mathbf{B}$ bisects $\mathbf{P}_{L}$ and $\mathbf{P}_{R}$.

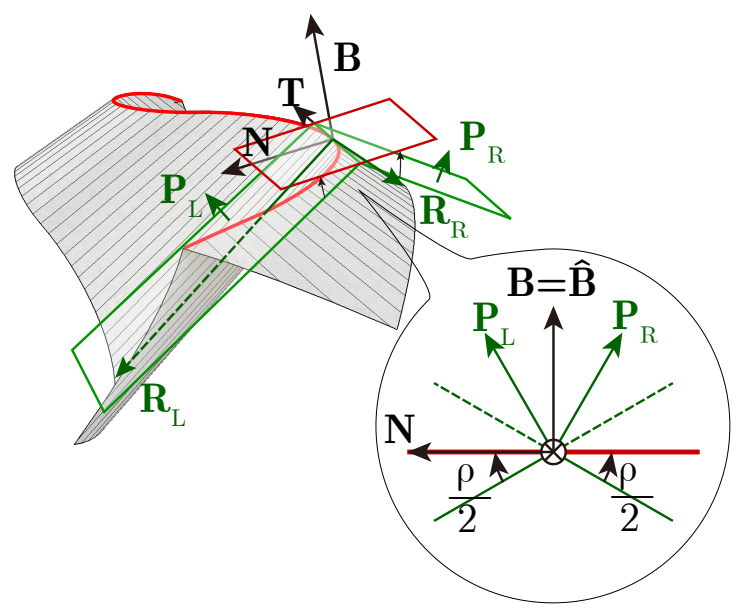

FIGURE 4. Binormal vector bisects surface normals.

4.2. Top-Side Frenet Frame. By Lemma 8 at $C^{2}$ cone-free points $\mathbf{X}(s)$, we can define the top-side normal of the osculating plane $\hat{\mathbf{B}}= \pm \mathbf{B}= \pm \mathbf{T} \times \mathbf{N}$ whose sign is defined such that $\hat{\mathbf{B}} \cdot \mathbf{P}_{L}=\hat{\mathbf{B}} \cdot \mathbf{P}_{R}>0$. Thus $\hat{\mathbf{B}}$ consistently points to the front side of the surface. By contrast, B's orientation depends on whether the $2 \mathrm{D}$ curve locally turns left or right (given by the sign of $k(s)$ ), flipping orientation at inflection points (where $k(s)=0$ ).

More formally, we will use the top-side Frenet frame given by $(\mathbf{T}(s), \hat{\mathbf{N}}(s), \hat{\mathbf{B}}(s))$ where $\hat{\mathbf{N}}(s)=\hat{\mathbf{B}}(s) \times \mathbf{T}(s)$.

Lemma 9. Consider a folded curved crease $\mathbf{X}(s)$ that is cone-free at a semikink $s=\tilde{s}$. The top-side Frenet frames are identical in positive and negative limits:

$$
\lim _{s \rightarrow \tilde{s}^{+}}(\mathbf{T}(s), \hat{\mathbf{N}}(s), \hat{\mathbf{B}}(s))=\lim _{s \rightarrow \tilde{s}^{-}}(\mathbf{T}(s), \hat{\mathbf{N}}(s), \hat{\mathbf{B}}(s))
$$

and thus the top-side Frenet frame is continuous at $s=\tilde{s}$. 
Proof. First, $\mathbf{T}(\tilde{s})$ is continuous because $\mathbf{X}(s)$ is $C^{1}$ at a semikink $s=\tilde{s}$.

Second, by Lemma 8 in the positive and negative limits, $\mathbf{B}(s)$ bisects $\mathbf{P}_{L}(s)$ and $\mathbf{P}_{R}(s)$. Because there is no cone ruling at $s=\tilde{s}$, the left and right surface normals $\mathbf{P}_{L}(s)$ and $\mathbf{P}_{R}(s)$ have equal positive and negative limits at $\tilde{s}$, so $\mathbf{P}_{L}(\tilde{s})$ and $\mathbf{P}_{R}(\tilde{s})$ are continuous. Thus $\mathbf{B}\left(\tilde{s}^{+}\right)$and $\mathbf{B}\left(\tilde{s}^{-}\right)$must lie on a common bisecting line of $\mathbf{P}_{L}(\tilde{s})$ and $\mathbf{P}_{R}(\tilde{s})$, and $\hat{\mathbf{B}}(\tilde{s})$ is uniquely defined by having positive dot product with $\mathbf{P}_{1}(\tilde{s})$ and $\mathbf{P}_{2}(\tilde{s})$. This gives us a unique definition of $\hat{\mathbf{B}}(s)$.

Third, $\hat{\mathbf{N}}(s)$ is continuous as $\hat{\mathbf{B}}(s) \times \mathbf{T}(s)$. Therefore $(\mathbf{T}(s), \hat{\mathbf{N}}(s), \hat{\mathbf{B}}(s))$ is continuous at $s=\tilde{s}$.

At $C^{2}$ points $X(s)$, we can define the signed curvature $\hat{K}(s)$ to flip sign where $\hat{\mathbf{N}}(s)$ does: $\hat{K}(s) \hat{\mathbf{N}}(s)=K(s) \mathbf{N}(s)$. As in $2 \mathrm{D}, \hat{K}(s)$ is positive where the curve turns left and negative where the curve turns right (relative to the top side).

4.3. General Bisection Property. By combining Lemmas 8 and 9 , we obtain a stronger bisection lemma:

Corollary 10. For a cone-free folded curved crease $\mathbf{X}(s), \hat{\mathbf{B}}(s)$ bisects $\mathbf{P}_{L}(s)$ and $\mathbf{P}_{R}(s)$. In particular, the tangent planes of the surfaces on both sides of $\mathbf{X}(s)$ form the same angle with the osculating plane.

4.4. Consequences. Using the bisector property, we can prove the nonexistence of a few strange situations.

Lemma 11. A crease $\mathbf{X}$ curved at $s$ cannot have a positive-length interval $s \in$ $(s-\varepsilon, s+\varepsilon)$ incident to a planar region.

Proof. If this situation were to happen, then the osculating plane of the curve must equal the plane of the planar region, which is say the left surface plane. By Corollary 10 the right surface plane must be the same plane. But then the folded piece of paper is actually planar along the crease, contradicting that it is not $C^{1}$ along the crease.

Lemma 12. A rule segment cannot be tangent to a cone-free curved crease point (at a relative interior point, in $2 D$ or $3 D$ ).

Proof. Suppose by symmetry that a rule segment is tangent to a crease point on its left side. If a rule segment is tangent to the crease point $\mathbf{x}(s)$ in $2 \mathrm{D}$, then it must also be tangent to $\mathbf{X}(s)$ in $3 \mathrm{D}$. There are two cases: (1) the left surface is a tangent surface generated from the crease; $(2)$ the surface is trimmed by the crease and is only tangent at the point $\mathbf{X}(s)$.

In Case 1, there is a finite portion of the crease that is $C^{2}$ and tangent to the incident rule segment. Then, for that portion of the crease (including $s$ ), the tangent plane of the left surface is the osculating plane of the curve.

In Case 2, consider surface normal $\mathbf{P}_{L}(s)$ at $\mathbf{X}(s)$. By assumption, the tangent vector $\mathbf{T}$ is parallel to the rule segment incident to $\mathbf{X}(s)$. Suppose by symmetry that $\mathbf{T}$ is actually the direction of the rule segment from $\mathbf{X}(s)$. (Otherwise, we could invert the parameterization of $\mathbf{X}$.) Because the surface normal is constant along the rule segment, and thus in the rule-segment direction, we have

$$
\frac{d \mathbf{P}_{L}}{d s^{+}}=\mathbf{0}
$$


Because $\mathbf{P}_{L}$ and $\mathbf{T}$ are perpendicular, $\frac{d}{d s^{+}}\left(\mathbf{P}_{L} \cdot \mathbf{T}\right)=0$, which expands to

$$
\frac{d \mathbf{P}_{L}}{d s^{+}} \cdot \mathbf{T}+\mathbf{P}_{L} \cdot \frac{d \mathbf{T}}{d s^{+}}=0 .
$$

Thus we obtain $\mathbf{P}_{L} \cdot \frac{d \mathbf{T}}{d s^{+}}=0$. Because the folded crease is not straight (Lemma 3), $\mathbf{N}$ is perpendicular to $\mathbf{P}_{L}$. Therefore the left tangent plane equals the osculating plane.

By Corollary 10, in either case, the right tangent plane must also equal the osculating plane, meaning that the folded piece of paper is actually planar along the crease, contradicting that it is not $C^{1}$ along the crease.

When the crease is $C^{2}$, Lemma 12 also implicitly follows from the Fuchs-Tabachnikov relation between fold angle and rule-segment angle Fuchs and Tabachnikov 99, Fuchs and Tabachnikov 07.

Corollary 13. For a crease $\mathbf{X}$ curved and cone-free at $s$, the point $\mathbf{X}(s)$ has an incident positive-length rule segment on the left side of $\mathbf{X}$ and an incident positivelength rule segment on the right side of $\mathbf{X}$.

Proof. First, by Lemma 11, $\mathbf{X}(s)$ is not locally surrounded by a flat region on either side, so by Corollary $5, \mathbf{X}(s)$ must have a rule segment on its left and right sides. Furthermore, such a rule segment cannot be a zero-length limit of nearby rule segments, because such a rule segment would be tangent to the curve, contradicting Lemma 12 .

Corollary 14. If a face's boundary is a $C^{1}$ curved closed curve, then the folded face's boundary is not $C^{1}$.

Proof. Consider the decomposition from Corollary 5 applied to the face, resulting in planar and ruled regions. By Lemma 11 , the ruled regions' boundary collectively cover the face boundary. The planar regions form a laminar (noncrossing) family in the face, so there must be a ruled region adjacent to only one planar region (or zero if the entire folded face is ruled). This ruled region is either the entire folded face or bounded by a portion of the face boundary and by a single rule segment (bounding a planar region). For each rule segment in the ruled region, we can discard the side that (possibly) contains the boundary rule segment, effectively shrinking the rule region while preserving its boundary structure of partial face boundary and one rule segment. In the limit of this process, we obtain a rule segment that is tangent to the face boundary. By Lemma 12 , this situation can happen only if the face is cone ruled at some point, which by Theorem 15 implies that the folded face boundary is not $C^{1}$.

\section{Smoоth Folding}

A smoothly folded crease is a folded crease that is $C^{1}$, i.e., kink-free. In Corollary 20 below, we will show that a smoothly folded crease is furthermore $C^{2}$, i.e., it cannot have semikinks. A smooth folding of a crease pattern is a folding in which every crease is smoothly folded. In this section, we characterize smooth folding as cone-free.

Theorem 15. If a folded crease $\mathbf{X}$ has a cone ruling at a point $\mathbf{X}(s)$, then $\mathbf{X}$ is kinked at $s$. 
Proof. Assume by symmetry that $\mathbf{X}(s)$ has a cone ruling on the left side, say clockwise from rule vector $\mathbf{R}_{1}$ to rule vector $\mathbf{R}_{2}$. Because the unfolded crease $\mathbf{x}$ is $C^{1}$, it has a tangent vector $\mathbf{t}$, so the left side of $\mathbf{x}(s)$ is, to the first order, the cone clockwise from $-\mathbf{t}$ to $\mathbf{t}$. Thus we have $-\mathbf{t}, \mathbf{r}_{1}, \mathbf{r}_{2}$, and $\mathbf{t}$ appearing in clockwise order around $\mathbf{x}(s)$, giving us the angle relation:

$$
180^{\circ}=\angle(-\mathbf{t}, \mathbf{t})=\angle\left(-\mathbf{t}, \mathbf{r}_{1}\right)+\angle\left(\mathbf{r}_{1}, \mathbf{r}_{2}\right)+\angle\left(\mathbf{r}_{2}, \mathbf{t}\right) .
$$

Now assume for contradiction that $\mathbf{X}$ is $C^{1}$ at $s$, so we can define the tangent vector $\mathbf{T}(s)$. By triangle inequality on the sphere, we have

$$
180^{\circ}=\angle(-\mathbf{T}, \mathbf{T}) \leq \angle\left(-\mathbf{T}, \mathbf{R}_{1}\right)+\angle\left(\mathbf{R}_{1}, \mathbf{R}_{2}\right)+\angle\left(\mathbf{R}_{2}, \mathbf{T}\right) .
$$

The latter three 3D angles must be smaller or equal to than the corresponding angles in $2 \mathrm{D}$, by isometry. Furthermore, $\angle\left(R_{1}, R_{2}\right)<\angle\left(\mathbf{r}_{1}, \mathbf{r}_{2}\right)$, because the surface must be bent along the entire cone ruling (otherwise it would have a flat patch). Therefore

$$
\angle\left(-\mathbf{T}, \mathbf{R}_{1}\right)+\angle\left(\mathbf{R}_{1}, \mathbf{R}_{2}\right)+\angle\left(\mathbf{R}_{2}, \mathbf{T}\right)<\angle\left(-\mathbf{t}, \mathbf{r}_{1}\right)+\angle\left(\mathbf{r}_{1}, \mathbf{r}_{2}\right)+\angle\left(\mathbf{r}_{2}, \mathbf{t}\right)=180^{\circ},
$$

a contradiction.
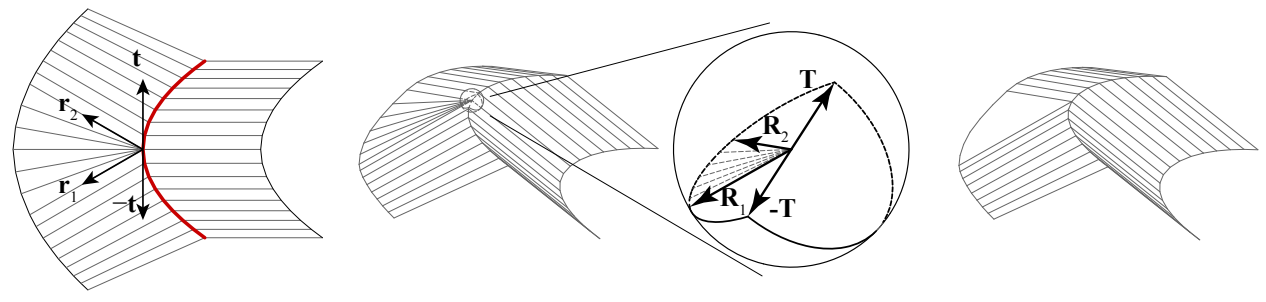

Figure 5. Cone rulings must fold into a kink in 3D.

Now we get a characterization of smooth folding:

Corollary 16. A folded curved crease $\mathbf{X}$ is kinked at $s$ if and only if it has a cone ruling at $\mathbf{X}(s)$.

Proof. Theorem 15 proves the "if" implication.

To prove the converse, consider a cone-free crease point $\mathbf{X}(s)$. In $2 \mathrm{D}$, we have a $180^{\circ}=\angle(-\mathbf{t}, \mathbf{t})$ angle on either side of the crease. We claim that this $180^{\circ}$ angle between the backward tangent and forward tangent is preserved by the folding, so the folded crease $\mathbf{X}$ has a continuous tangent and thus is $C^{1}$ at $s$.

First, suppose that there is no planar region incident to $\mathbf{X}(s)$ on say the left side. Then the left side is locally a uniquely ruled $C^{2}$ surface, with no rule segments tangent to the curve by Lemma 12 , and thus the surface can be extended slightly to include $\mathbf{X}(s)$ in its interior. In a $C^{1}$ surface, it is known that geodesic (2D) angles equal Euclidean (3D) angles, so folding preserves the $180^{\circ}$ angle between the backward and forward tangents.

Now suppose that there is a planar region on the left side of $\mathbf{X}(s)$. By Lemma 6 . there can be only one, and by Lemma 11 , there must be two uniquely ruled surfaces separating such a planar region from the crease. These three surfaces meet smoothly with a common surface normal, as the surface is $C^{2}$ away from the crease, so the overall angle between the backward and forward tangents of the crease equals the 
sum of the three angles of the surfaces at $\mathbf{X}(s)$. The previous paragraph argues that the two uniquely ruled surfaces preserve their angles, and the planar region clearly preserves its angle (it is not folded). Hence, again, folding preserves the $180^{\circ}$ angle between the backward and forward tangents.

\section{Mountains and Valleys}

6.1. Crease. Refer to Figure 4. For a smoothly folded (cone-free) crease $\mathbf{X}$, the fold angle $\rho \in\left(-180^{\circ}, 180^{\circ}\right)$ at $\mathbf{X}(s)$ is defined by $\cos \rho=\mathbf{P}_{L} \cdot \mathbf{P}_{R}$ and $\sin \rho=\left[\left(\mathbf{P}_{L} \times\right.\right.$ $\left.\left.\mathbf{P}_{R}\right) \cdot \mathbf{T}\right]$. The crease is valley at $s$ if the fold angle is negative, i.e., $\left(\mathbf{P}_{L} \times \mathbf{P}_{R}\right) \cdot \mathbf{T}<0$. The crease is mountain at $s$ if the fold angle is positive, i.e., $\left(\mathbf{P}_{L} \times \mathbf{P}_{R}\right) \cdot \mathbf{T}>0$.

Lemma 17. A smoothly folded curved crease $\mathbf{X}$ has a continuous fold angle $\rho \neq 0$.

Proof. By Corollary 16 , the crease is cone-free, so the surface normals $\mathbf{P}_{L}(s)$ and $\mathbf{P}_{R}(s)$ are continuous. If the resulting fold angle $\rho(s)$ were zero, then we would have $\mathbf{P}_{L}(s)=\mathbf{P}_{R}(s)$, contradicting that the folded piece of paper is not $C^{1}$ at crease point $\mathbf{X}(s)$.

Corollary 18. A smoothly folded curved crease $\mathbf{X}$ is mountain or valley throughout.

Proof. By Lemma 17, $\rho(s)$ is continuous and nonzero. By the intermediate value theorem, $\rho(s)$ cannot change sign.

Lemma 19. For a smoothly folded curved crease $\mathbf{X}(s)$,

$$
\hat{K}(s) \cos \frac{1}{2} \rho(s)=\hat{k}(s) .
$$

In particular, folding increases curvature: $|\hat{k}(s)|<|\hat{K}(s)|$, i.e., $k(s)<K(s)$.

Proof. Referring to Figure 4, we have

$$
\cos \frac{1}{2} \rho(s)=\mathbf{P}_{L}(s) \cdot \hat{\mathbf{B}}(s) .
$$

By definition of $\hat{\mathbf{B}}(s)$, this dot product is the triple product

$$
\mathbf{P}_{L}(s) \cdot(\mathbf{T}(s) \times \hat{\mathbf{N}}(s))=\hat{\mathbf{N}}(s) \cdot\left(\mathbf{P}_{L}(s) \times \mathbf{T}(s)\right)
$$

(similar to the proof of Lemma 8). Multiplying by $\hat{K}(s)$, we obtain

$$
(\hat{K}(s) \hat{\mathbf{N}}(s)) \cdot\left(\mathbf{P}_{L}(s) \times \mathbf{T}(s)\right)=(K(s) N(s)) \cdot\left(\mathbf{P}_{L}(s) \times \mathbf{T}(s)\right) .
$$

By Lemma 7 , this geodesic curvature is $\hat{\mathbf{k}}(s)$.

Corollary 20. A folded crease cannot have a semikink, and thus a smoothly folded crease $\mathbf{X}$ is $C^{2}$.

Proof. Suppose $\mathbf{X}(s)$ had a semikink at $s=\tilde{s}$. Applying Lemma 19 with positive and negative limits, we obtain that

$$
\lim _{s \rightarrow \tilde{s}^{+}} \hat{K}(s)=\frac{\hat{k}(s)}{\cos \frac{1}{2} \rho}=\lim _{s \rightarrow \tilde{s}^{-}} \hat{K}(s),
$$

and thus the signed curvature $\hat{K}(s)$ is continuous at $s=\tilde{s}$. By Lemma $9 \hat{\mathbf{N}}(s)$ is continuous at $s=\tilde{s}$. Therefore $\frac{d^{2} \mathbf{X}(s)}{d s^{2}}=\hat{K}(s) \hat{\mathbf{N}}(s)$ is continuous at $s=\tilde{s}$, so $\mathbf{X}(\tilde{s})$ is not actually a semikink. 
Lemma 21. A smoothly folded crease $\mathbf{X}$ is valley if and only if $\left(\mathbf{P}_{L} \times \hat{\mathbf{B}}\right) \cdot \mathbf{T}<0$, and mountain if and only if $\left(\mathbf{P}_{L} \times \hat{\mathbf{B}}\right) \cdot \mathbf{T}>0$.

Proof. Refer to Figure 4 Vectors $\mathbf{P}_{L}, \mathbf{P}_{R}$, and $\hat{\mathbf{B}}$ are all perpendicular to $\mathbf{T}$, and thus live in a common oriented plane with normal $\mathbf{T}$. By the choice of $\hat{\mathbf{B}}$ to have positive dot products with $\mathbf{P}_{L}$ and $\mathbf{P}_{R}$, the three vectors in fact live in a common half-plane. In this plane, we can see the fold angle $\rho=\angle\left(\mathbf{P}_{L}, \mathbf{P}_{R}\right)$, where $\angle$ measures the convex angle between the vectors, signed positive when the angle is convex in the counterclockwise orientation within the oriented plane with normal $\mathbf{T}$, and signed negative when clockwise.

By Corollary 10, $\mathbf{P}_{L} \cdot \mathbf{B}=\mathbf{P}_{R} \cdot \mathbf{B}$, so $\mathbf{P}_{L} \cdot \hat{\mathbf{B}}=\mathbf{P}_{R} \cdot \hat{\mathbf{B}}$. Thus $\cos \angle\left(\mathbf{P}_{L}, \hat{\mathbf{B}}\right)=$ $\cos \angle\left(\mathbf{P}_{R}, \hat{\mathbf{B}}\right)$, i.e., $\left|\angle\left(\mathbf{P}_{L}, \hat{\mathbf{B}}\right)\right|=\left|\angle\left(\mathbf{P}_{R}, \hat{\mathbf{B}}\right)\right|$.

If $\angle\left(\mathbf{P}_{L}, \hat{\mathbf{B}}\right)=\angle\left(\mathbf{P}_{R}, \hat{\mathbf{B}}\right)$, then $\mathbf{P}_{L}=\mathbf{P}_{R}$, contradicting that $\mathbf{X}$ is a crease. Therefore, $\angle\left(\mathbf{P}_{L}, \hat{\mathbf{B}}\right)=\angle\left(\hat{\mathbf{B}}, \mathbf{P}_{R}\right)= \pm \frac{1}{2} \angle\left(\mathbf{P}_{L}, \mathbf{P}_{R}\right)$. Because $\left|\angle\left(\mathbf{P}_{L}, \hat{\mathbf{B}}\right)\right|<90^{\circ}$, we must in fact have $\angle\left(\mathbf{P}_{L}, \hat{\mathbf{B}}\right)=\angle\left(\hat{\mathbf{B}}, \mathbf{P}_{R}\right)=\frac{1}{2} \angle\left(\mathbf{P}_{L}, \mathbf{P}_{R}\right)$, i.e., $\hat{\mathbf{B}}$ bisects the convex angle $\angle\left(\mathbf{P}_{L}, \mathbf{P}_{R}\right)$. Hence $\hat{\mathbf{B}}$ lies in between $\mathbf{P}_{L}$ and $\mathbf{P}_{R}$ within the halfplane. Therefore the cross products $\mathbf{P}_{L} \times \mathbf{P}_{R}, \mathbf{P}_{L} \times \hat{\mathbf{B}}$, and $\hat{\mathbf{B}} \times \mathbf{P}_{R}$ are all parallel, so their dot products with $\mathbf{T}$ have the same sign.

6.2. Rule Segment. We can also define whether a rule segment bends the paper mountain or valley; refer to Figure6. Consider a relative interior point $\mathbf{Y}$ of a rule segment with direction vector $\mathbf{R}$, with top-side surface normal $\mathbf{P}$. Then we can construct a local Frenet frame at $\mathbf{Y}$ with tangent vector $\mathbf{Q}=\mathbf{R} \times \mathbf{P}$, normal vector $\mathbf{P}$, and binormal vector $\mathbf{R}$. These frames define a $3 \mathrm{D}$ curve $\mathbf{Y}(t)$ where $\mathbf{Y}(0)=\mathbf{Y}$, which follows the principle curvature of the surface. Parameterize this curve by arc length.
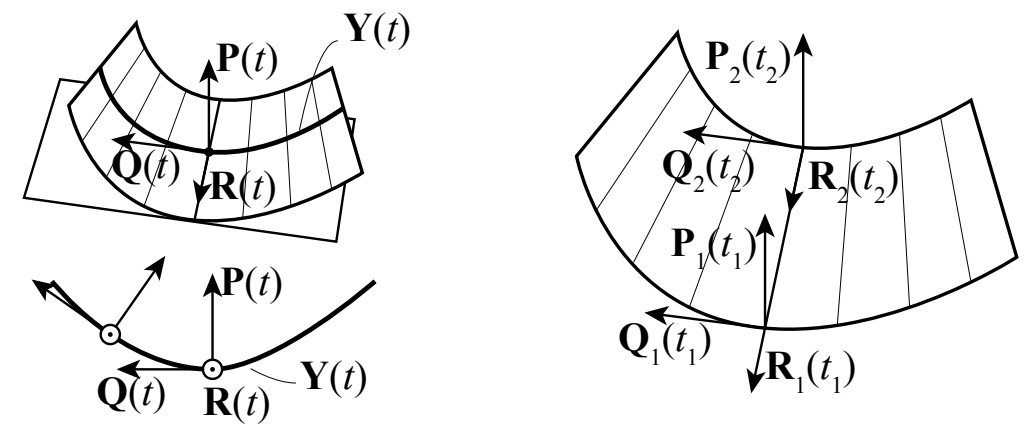

Figure 6. Defining a frame around an interior point to define mountain vs. valley bending.

First consider the case when the surface is $C^{2}$ at $Y(t)$. The surface bends valley at $\mathbf{Y}(t)$ if the curvature vector $\frac{d^{2} \mathbf{Y}(t)}{d t^{2}}=\frac{d \mathbf{Q}(t)}{d t}$ is on the top side, i.e., has positive dot product with $\mathbf{P}(t)$; and it bends mountain if $\frac{d \mathbf{Q}(t)}{d t} \cdot \mathbf{P}(t)<0$. In particular, at $t=0$, we determine whether the original rule segment bends mountain or valley at $\mathbf{Y}$.

If the surface is not $C^{2}$ at $Y(t)$, then the rule segment is a semicrease, which connects two $C^{2}$ surfaces sharing a surface normal at the crease; refer to Figure 7 . 
In this case, the surface bends valley at $\mathbf{Y}(t)$ when the two surfaces bends valley; or one of the surface is planar, and the other bends valley. Similarly, the surface bends mountain at $\mathbf{Y}(t)$ when the two surfaces bends mountain; or one of the surface is planar, and the other bends mountain. At an inflection point, there is no mountain/valley assignment.
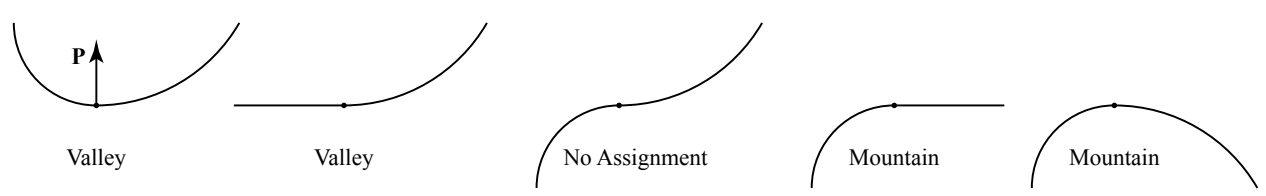

FiguRE 7. Definition of mountain and valley for a semicrease.

Lemma 22. A developable uncreased surface bends the same direction (mountain or valley) at every relative interior point of a rule segment.

Proof. First consider the case when the surface is $C^{2}$. Consider two points $\mathbf{Y}_{1}$ and $\mathbf{Y}_{2}$ on the rule segment, with principle curvature frames $\left(\mathbf{Q}_{i}\left(t_{i}\right), \mathbf{R}_{i}\left(t_{i}\right), \mathbf{P}_{i}\left(t_{i}\right)\right)$. Choose $t_{2}$ as a function of $t_{1}$ so that $\mathbf{Y}_{1}\left(t_{1}\right)$ and $\mathbf{Y}_{2}\left(t_{2}\right)$ lie on a common rule segment. Then the frames are in fact identical: $\mathbf{R}_{1}\left(t_{1}\right)=\mathbf{R}_{2}\left(t_{2}\right)$ is the common rule direction, $\mathbf{P}_{1}\left(t_{1}\right)=\mathbf{P}_{2}\left(t_{2}\right)$ is the common top-side surface normal, and $\mathbf{Q}_{1}\left(t_{1}\right)=$ $\mathbf{Q}_{2}\left(t_{2}\right)$ is their cross product. Because the surface is locally $C^{2}$ around the ruled segment $\mathbf{Y}_{1}$ and $\mathbf{Y}_{2}$, we have $\frac{d t_{2}}{d t_{1}}>0$, so

$$
\frac{d \mathbf{Q}_{2}\left(t_{2}\right)}{d t_{2}} \cdot \mathbf{P}=\frac{d \mathbf{Q}_{1}\left(t_{1}\right)}{d t_{2}} \cdot \mathbf{P}=\frac{d t_{2}}{d t_{1}} \frac{d \mathbf{Q}_{1}\left(t_{1}\right)}{d t_{1}} \cdot \mathbf{P} .
$$

Therefore, the surface bends the same direction.

Next consider the case when the surface is not $C^{2}$, i.e., the rule segment is a semicrease between $C^{2}$ surfaces $S^{+}$and $S^{-}$. By the above argument, in a $C^{2}$ patch, the inflection occurs along the rule segment where $\frac{d \mathbf{Q}(t)}{d t} \cdot \mathbf{P}=0$ is satisfied. Also, if surface is not $C^{2}$, then it is on a rule segment. Therefore, if the $S^{-}$surface is bent in a different direction at $\lim _{t \rightarrow t_{1}^{-}} \mathbf{Y}_{1}(t)$ and $\lim _{t \rightarrow t_{2}^{-}} \mathbf{Y}_{2}\left(t_{2}\right)$, then a path from $\mathbf{Y}_{1}$ to $\mathbf{Y}_{2}$ must cross a rule segment. Because rule segments do not intersect, $S^{+}$and $S^{-}$keeps their own bending orientations. Therefore, the assignment for the semicrease is unchanged along the segment.

By Lemma 22, we can define the bending direction of a rule segment: a developable uncreased surface bends mountain or valley at a rule segment if a relative interior point of the rule segment bends mountain or valley, respectively. Furthermore, because the frames are identical, we can define the principle curvature frame $(\mathbf{Q}, \mathbf{R}, \mathbf{P})$ of a rule segment by the principle curvature frame at any relative interior point on the rule segment.

6.3. Crease vs. Rule Segment. Next we consider the mountain-valley relation between a rule segment and a crease.

First consider a smoothly folded crease $\mathbf{X}$ with left and right surface ruling vectors $\mathbf{R}_{L}$ and $\mathbf{R}_{R}$, defined as unit vectors which lie along the rule segments on surfaces $S_{L}$ and $S_{R}$ incident to $\mathbf{X}$. (If there is a planar region incident to $\mathbf{X}$, these 
ruling vectors will not be unique.) A left-side ruling vector $\mathbf{R}_{L}$ lives in the plane perpendicular to $\mathbf{P}_{L}$. Therefore, the vector can be represented by

$$
\mathbf{R}_{L}=\left(\cos \theta_{L}\right) \mathbf{T}+\left(\sin \theta_{L}\right)\left(\mathbf{P}_{L} \times \mathbf{T}\right),
$$

where we call $\theta_{L}$ the left-side ruling angle of the ruling, which is nonzero by Lemma 12. Because the ruling angle is intrinsic, the ruling vector in $2 \mathrm{D}$ is represented by $\mathbf{r}_{L}=\left(\cos \theta_{L}\right) \mathbf{t}+\left(\sin \theta_{L}\right) \hat{\mathbf{b}}$. The orientation of the left-side ruling vector is chosen to orient to the left, i.e., $\mathbf{r}_{L} \cdot \hat{\mathbf{b}}>0$, so $\theta_{L}$ is positive. Similarly, ruling vector $\mathbf{R}_{R}$ on the right surface is represented by $\mathbf{R}_{R}=\left(\cos \theta_{R}\right) \mathbf{T}-\left(\sin \theta_{R}\right)\left(\mathbf{P}_{R} \times \mathbf{T}\right)$, using right-side ruling angle $\theta_{R}$. The orientation is chosen to be on the right side, so $\theta_{R}>0$.

Lemma 23. Consider a uniquely ruled smoothly folded crease $\mathbf{X}$ with locally $C^{2}$ surfaces on both sides (no semicreases). Then the rule segment on the left side of $\mathbf{X}$ bends valley if and only if $\mathbf{N} \cdot \mathbf{P}_{L}>0$. Symmetrically, the surface bends valley on the right side if and only if $\mathbf{N} \cdot \mathbf{P}_{R}>0$.

Proof. Build the principle curvature frame $(\mathbf{Q}(t), \mathbf{R}(t), \mathbf{P}(t))$ of rule segment parameterized by the arclength $t$ in the principle curvature direction. Consider corresponding point $\mathbf{X}(s)$ and the arclength parameter $s=s(t)$ along the crease at the rule segment parameterized by $t$. Because the surface is locally $C^{2}$ around the rule segment, $\frac{d s}{d t}>0$. Because we consider the left side of the surface, $\mathbf{P}_{L}(s)=\mathbf{P}(t)$. Let $\theta$ be the angle between $\mathbf{R}(t)$ and $\mathbf{T}(s)$, i.e., $\mathbf{T}(s)=\sin \theta \mathbf{Q}(t)+\cos \theta \mathbf{R}(t)$. By Lemma $12,0<\theta<\pi$, and we get

$$
\mathbf{Q}=(\csc \theta) \mathbf{T}-(\cot \theta) \mathbf{R} .
$$

Assume that the surface bends valley at the rule segment, i.e.,

$$
V(t)=\frac{d \mathbf{Q}(t)}{d t} \cdot \mathbf{P}(t)>0
$$

Using orthogonality of vectors $\mathbf{Q}$ and $\mathbf{P}$, i.e., $\mathbf{Q}(t) \cdot \mathbf{P}(t)=0$, and taking derivatives, we obtain

$$
\frac{d \mathbf{Q}}{d t} \cdot \mathbf{P}+\mathbf{Q} \cdot \frac{d \mathbf{P}}{d t}=0
$$

Then,

$$
\begin{aligned}
V(t) & =-\mathbf{Q} \cdot \frac{d \mathbf{P}}{d t} \\
& =-((\csc \theta) \mathbf{T}-(\cot \theta) \mathbf{R}) \cdot \frac{d \mathbf{P}}{d t} \\
& =-(\csc \theta) \mathbf{T} \cdot \frac{d \mathbf{P}}{d t} .
\end{aligned}
$$

Here, we used Equation 1. By the orthogonality of vectors $\mathbf{T}$ and $\mathbf{P}$, we get

$$
\mathbf{T} \cdot \frac{d \mathbf{P}}{d t}=\frac{d \mathbf{T}}{d t} \cdot \mathbf{P}
$$


Then,

$$
\begin{aligned}
V(t) & =(\csc \theta) \frac{d \mathbf{T}(s)}{d t} \cdot \mathbf{P}(t) \\
& =(\csc \theta) \frac{d s}{d t} \frac{d \mathbf{T}(s)}{d s} \cdot \mathbf{P}(t) \\
& =(\csc \theta) \frac{d s}{d t} K(s) \mathbf{N}(s) \cdot \mathbf{P}_{L}(s)
\end{aligned}
$$

Because $\csc \theta>0, \frac{d s}{d t}>0$, and $K(s)>0$, Equation 2 is equivalent to $\mathbf{N}(s) \cdot \mathbf{P}_{L}(s)>$ 0 .

Now we make a stronger statement, allowing the ruling vectors to be not unique and the surfaces to be not $C^{2}$.

Corollary 24. Consider a smoothly folded crease $\mathbf{X}$. Then a rule segment on the left side of $\mathbf{X}$ bends valley if and only if $\mathbf{N} \cdot \mathbf{P}_{L}>0$. Symmetrically, the surface bends valley on the right side if and only if $\mathbf{N} \cdot \mathbf{P}_{R}>0$.

Proof. Consider rule segments at $\mathbf{X}(\tilde{s})$. By Theorem 15 , the crease is cone free, so a rule segment is either (1) between two $C^{2}$ ruled surfaces, or (2) between a plane and a $C^{2}$ ruled surfaces.

Consider Case 1, and let $S^{-}$and $S^{+}$be the two surfaces. Because there are no cone rulings, $S^{-}$and $S^{+}$are locally formed by unique rulings emanating from $\mathbf{X}(s)$ at $s<\tilde{s}$ and $s>\tilde{s}$, respectively. Then

$$
\lim _{s \rightarrow \tilde{s}^{-}} \mathbf{N}(s) \cdot \mathbf{P}_{L}(s)=\lim _{s \rightarrow \tilde{s}^{+}} \mathbf{N}(s) \cdot \mathbf{P}_{L}(s)=\mathbf{N}(s) \cdot \mathbf{P}_{L}(s) .
$$

So both surfaces $S^{-}$and $S^{+}$bend valley if and only if $\mathbf{N}(s) \cdot \mathbf{P}_{L}(s)>0$.

Next consider Case 2. By symmetry, assume that $S^{-}$is planar and $S^{+}$is $C^{2}$ ruled surface. Then $S^{+}$is locally formed by unique rule segments emanating from $\mathbf{X}(s)$ at $s>\tilde{s}$. Hence $S^{+}$, and thus the rule segment, bends valley if and only if $\mathbf{N}(s) \cdot \mathbf{P}_{L}(s)>0$.

Theorem 25. Consider a smoothly folded curved crease $\mathbf{X}$. A rule segment incident to $\mathbf{X}(\tilde{s})$ on the convex side of $\mathbf{X}(\tilde{s})$ has the same mountain/valley assignment as the crease, while a rule segment incident to $\mathbf{X}(\tilde{s})$ on the concave side of $\mathbf{X}(\tilde{s})$ has the opposite mountain/valley assignment as the crease.

Proof. Assume by symmetry that the left side of the paper is the convex side $(\hat{k}(s)<$ $0)$. Also, assume that the crease is a valley, i.e., $\left(\hat{\mathbf{B}} \times \mathbf{P}_{L}\right) \cdot \mathbf{T}=\left(\mathbf{P}_{L} \times \mathbf{B}\right) \cdot \mathbf{T}>0$. Then the top-side normal of the osculating plane is $\hat{\mathbf{B}}=-\mathbf{B}$, and thus $\hat{\mathbf{N}}=-\mathbf{N}$.

Now

$$
\begin{aligned}
\left(\mathbf{P}_{L} \times \mathbf{B}\right) \cdot \mathbf{T} & =\left(\mathbf{P}_{L} \times(\mathbf{T} \times \mathbf{N})\right) \cdot \mathbf{T} \\
& =\left(\mathbf{T}\left(\mathbf{P}_{L} \cdot \mathbf{N}\right)-\mathbf{N}\left(\mathbf{P}_{L} \cdot \mathbf{T}\right)\right) \cdot \mathbf{T}>0 .
\end{aligned}
$$

The second term disappears because $\mathbf{P}_{L} \cdot \mathbf{T}=0$. Therefore $\mathbf{P}_{L} \cdot \mathbf{N}>0$, so the left side is valley. 


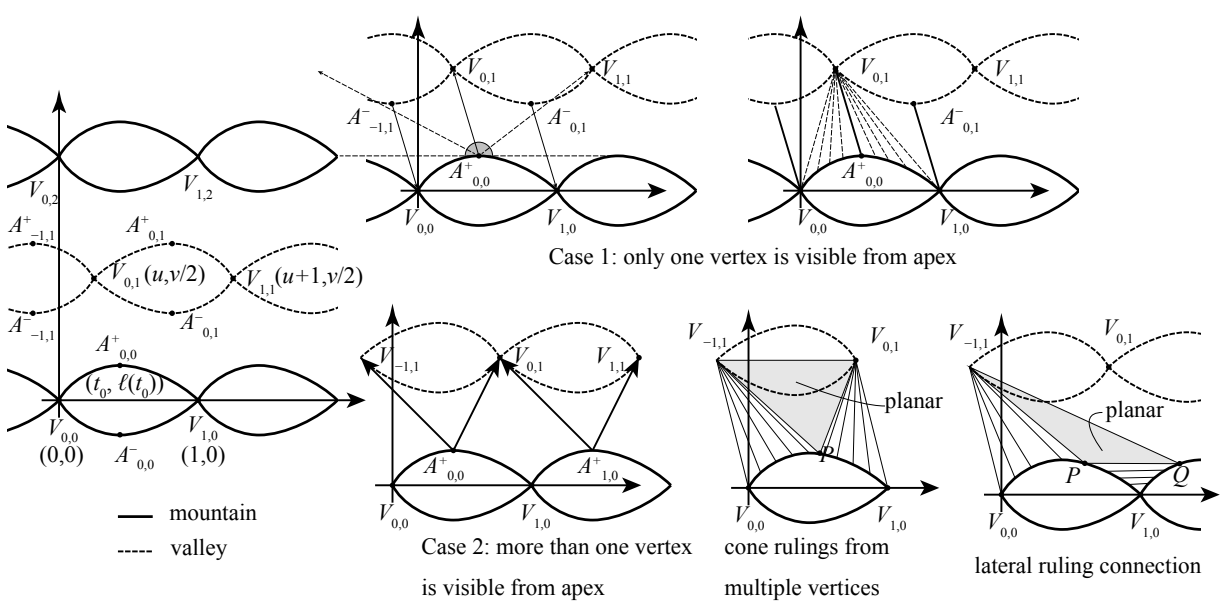

FiguRE 8. Ruling conditions for a lens tessellation.

6.4. Creases Connected by a Rule Segment. Now consider two creases connected by a rule segment. By Lemma 25, we get the following.

Corollary 26. Consider two smoothly folded creases connected by a rule segment. If the rule segment is on the concave sides of both creases, or on the convex sides of both creases, then the creases must have the same direction (mountain or valley). If a rule segment is on the convex side of one crease and the concave side of the other crease, then the creases must have the opposite direction (one mountain and one valley).

\section{Lens Tessellation}

In this section, we use the qualitative properties of rulings obtained in previous sections to reconstruct rule segments from a crease pattern of the generalized version of lens tessellation.

First, as illustrated in Figure 8, we define the lens tessellation parameterized by a convex $C^{2}$ function $\ell:[0,1] \rightarrow[0, \infty)$ with $\ell(0)=\ell(1)=0$, horizontal offset $u \in[0,1)$, and vertical offset $v \in(0, \infty)$, to consist of

(1) mountain creases $\gamma_{i, 2 j}^{ \pm}=\{(t+i, \pm \ell(t)+j v) \mid t \in[0,1]\}$ for $i, j \in \mathbb{Z}$; and

(2) valley creases $\left.\gamma_{i, 2 j+1}^{ \pm}=\left\{\left(1-t+i+u, \pm \ell(1-t)+\left(j+\frac{1}{2}\right) v\right)\right\} \mid t \in[0,1]\right\}$ for $i, j \in \mathbb{Z}$.

Define the vertices to be points of the form $V_{i, 2 j}=(i, j v)$ and $V_{i, 2 j+1}=(i+u,(j+$ $\left.\frac{1}{2}\right) v$ ). Four creases meet at each vertex.

Because $\ell(t)$ is convex, it has a unique maximum $\ell\left(t^{*}\right)$ at some $t=t^{*}$. Define the apex $A_{i, k}$ of crease $\gamma_{i, k}^{ \pm}$to be the point of the crease at $t=t^{*}$, i.e., $A_{i, 2 j}^{ \pm}=$ $\left(t^{*}+i, \pm \ell\left(t^{*}\right)+j v\right)$ and $A_{i, 2 j+1}^{ \pm}=\left(1-t^{*}+i+u, \pm \ell\left(1-t^{*}\right)+\left(j+\frac{1}{2}\right) v\right)$.

7.1. Necessary Conditions. Consider a crease point $\mathbf{x}(s)$. A point $\mathbf{y}$ on the crease pattern (a vertex or crease point) is visible from $\mathbf{x}(s)$ on the left (right) side of $\mathbf{x}$ at $\mathbf{x}(s)$ if the oriented open line segment $\overrightarrow{\mathbf{x}(s) \mathbf{y}}$ is on the left (right) side of $\mathbf{x}(s)$ 
and does not share a point with the crease pattern. If $\mathbf{x}(s)$ and $\mathbf{y}$ are the endpoints of a rule segment, then certainly they must be visible from each other.

Theorem 27. A lens tessellation can smoothly fold only if there is a vertex $V_{i, 1}$ visible from every point on crease $\gamma_{0,0}^{+}$on the convex side.

Proof. Refer to Figure 8, By Corollary 13 , there must be a rule segment emanating from $A_{0,0}^{+}$on the convex side of $\gamma_{0,0}^{+}$. The other endpoint $B$ of that rule segment must be visible from $A_{0,0}^{+}$on the convex side of $\gamma_{0,0}^{+}$. Because the tangent line of $\gamma_{0,0}^{+}$ at $A_{0,0}^{+}$is horizontal, any such visible point $B$ must lie on the union of creases $\gamma_{i, 1}^{-}$ and vertices $V_{i, 1}$ for $i \in \mathbb{Z}$. By Theorem 26, $B$ cannot be on the relative interior of one of the valley creases $\gamma_{i, 1}^{-}$because then the rule segment would be on the concave sides of creases of opposite direction. Thus $B$ must be among the vertices $V_{i, 1}$ for $i \in \mathbb{Z}$.

First consider the case that only one vertex $V_{n, 1}$ is visible from $A_{0,0}^{+}$on the convex side of $\gamma_{0,0}^{+}$. Then $A_{0,0}^{+} V_{n, 1}$ must be a rule segment. By symmetry, $V_{1,0} A_{n, 1}^{-}$ is also a rule segment. Consider a point on $\gamma_{0,0}^{+}$between $A_{0,0}^{+}$and $V_{0,1}$, which by Corollary 13 has a rule segment on the positive side of $\gamma_{0,0}^{+}$. This rule segment cannot cross the existing rule segments $A_{0,0}^{+} V_{n, 1}$ and $V_{1,0} A_{n, 1}^{-}$, so its other endpoint must be $V_{n, 1}, A_{n, 1}^{-}$, or between $V_{n, 1}$ and $A_{n, 1}^{-}$on curve $\gamma_{n, 1}^{-}$. By Theorem 26 , the only possible ruling is to have a cone apex at $V_{n, 1}$. Similarly, rule segments from points between $V_{0,0}$ and $A_{0,0}^{+}$on $\gamma_{0,0}^{+}$must end at $V_{n, 1}$. Therefore $V_{n, 1}$ is visible from every point on crease $\gamma_{0,0}^{+}$on the convex side.

Second consider the case in which more than one vertex $V_{i, 1}$ is visible from apex $A_{0,0}^{+}$on the convex side of $\gamma_{0,0}^{+}$. Suppose for contradiction that there is no common vertex visible from the entire curve $\gamma_{0,0}^{+}$. Similar to the previous case, there must be a rule segment from apex $A_{0,0}^{+}$to one of the vertices $V_{n, 1}$. But we assumed that some other point of $\gamma_{0,0}^{+}$cannot see $V_{n, 1}$. By symmetry, suppose that point is to the right of $A_{0,0}^{+}$. There is a transition point $P$ on the relative interior of $\gamma_{0,0}^{+}$when the endpoints of rulings change from $V_{n, 1}$ to either (a) another vertex $V_{m, 1}$ with $m>n$ or (b) a point on $\gamma_{1,0}^{+}$. (See Figure 8.) At such a point $P$, we have two rule segments. By Theorem 15, $P$ cannot be a cone apex. Hence there must be a planar region between the two rule segments. Specifically, in case (a), the triangle $P V_{n, 1} V_{m, 1}$ is planar, which contains all of $\gamma_{n, 1}^{-}$, contradicting that the folded piece of paper is not $C^{1}$ on $\gamma_{n, 1}^{-}$. In case (b), let $Q$ be the point on $\gamma_{1,0}^{+}$. The triangle $P Q V_{n, 1}$ is planar. This triangle cannot intersect $\gamma_{n, 1}^{-}$, because the folded piece of paper is not $C^{1}$ on $\gamma_{n, 1}^{-}$. In particular, the curve $\gamma_{n, 1}^{-}$cannot intersect the segment $V_{n, 1} V_{0,1}$ (which begins in the triangle). Because $\gamma_{1,0}^{+}$is a $180^{\circ}$ rotation of $\gamma_{n, 1}^{-}$ mapping $V_{n, 1}$ to $V_{0,1}$, we symmetrically have that the curve $\gamma_{1,0}^{+}$cannot intersect the same segment $V_{n, 1} V_{0,1}$. Thus this segment is a visibility segment, as is $V_{n, 1} V_{0,0}$. By convexity of the lens, $V_{n, 1}$ can see the entire curve $\gamma_{1,0}^{+}$. Therefore there is in fact a common vertex visible from the curve $\gamma_{0,0}^{+}$.

7.2. Existence / Sufficiency. Finally we prove that the condition from Theorem 27 is also sufficient:

Theorem 28. A lens tessellation can fold smoothly if there is a vertex $V_{i, 1}$ visible from every point on crease $\gamma_{0,0}^{+}$on the convex side. 


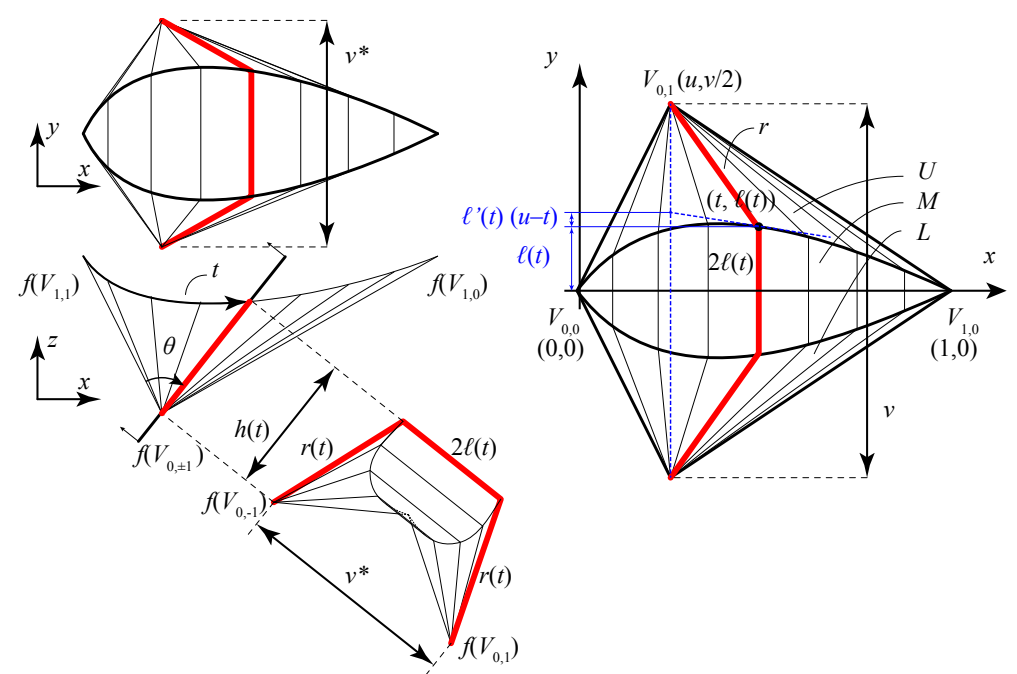

Figure 9. A modular kite structure.

Proof. First we construct the folding of one "gadget", $(i, j)=0$; refer to Figure 9 We can add an integer to $u$ to assume that $V_{0,1}$ is the visible vertex from apex $A_{0,0}^{+}$. In $2 \mathrm{D}$, this gadget is bounded by a quadrangle of rule segments with vertex coordinates $V_{0,0}=(0,0), V_{0,-1}=\left(u,-\frac{1}{2} v\right), V_{1,0}=(1,0), V_{0,1}=\left(u, \frac{1}{2} v\right)$. This kite module is decomposed by its creases into an upper wing part $U$, middle lens part $M$, and lower wing part $L$. We assume that $M$ is ruled parallel to $y$ axis: the rule segments of $M$ are of the form $(t, \ell(t))$ and $(t,-\ell(t))$ parameterized by $t$. (We can make this assumption because we are constructing a folded state.) We also assume that $U$ consists of cone rulings between $V_{0,1}$ and $(t, \ell(t))$ while $L$ consists of cone rulings between $V_{0,-1}$ and $(t,-\ell(t))$ using the same parameter $t$.

The folding $f(M)$ is a cylindrical surface with parallel rulings. We orient the folded form such that this ruling direction is parallel to $y$ axis and $\overrightarrow{f\left(V_{0,0}\right) f\left(V_{1,0}\right)}$ is parallel to the positive direction of $x$ axis. Then the orthogonal projection of $f(M)$ to $x z$ plane is a curve $\gamma$, and a ruling at $t$ on $M$ corresponds to a point on $\gamma(t)$ while $t$ is the arclength parameter.

We further assume that the folded state is symmetric with respect to reflection through a plane passing through $\overline{f\left(V_{0,0}\right) f\left(V_{1,0}\right)}$ and parallel to $x z$ plane. Let the distance between $f\left(V_{0,-1}\right)$ and $f\left(V_{0,1}\right)$ be denoted by $v^{*}$ where $0<v^{*}<v$. We will show that there is a valid folded state for arbitrary $v^{*}$ if it is sufficiently close to $v$.

Consider the set of rule segments of $U, M$, and $L$ at parameter $t$ and its folding. Then, by our symmetry assumption, these segments form a planar polyline which together with segments $f\left(V_{0,-1}\right)$ and $f\left(V_{0,1}\right)$ form an isosceles trapezoid with the base length $v^{*}$ and top length $2 \ell(t)$. The legs are the length of the rule segments, which can be calculated from the crease pattern as $r(t)=\sqrt{(u-t)^{2}+(v / 2-\ell(t))^{2}}$. Such a trapezoid exists because $0<v^{*}<v \leq 2 \ell(t)+2 r(t)$. The height of the trapezoid $h(t)$ is given by

$$
h(t)=\sqrt{\left(v-v^{*}\right)\left(\frac{v+v^{*}}{4}-\ell(t)\right)+(t-u)^{2}} .
$$


Now consider the projection of this trapezoid in $x z$ plane. This projection is a line segment between two points, namely the projections of $V_{0,1}$ and $\gamma(t)$, and it must have length of $h(t)$. We use the following lemma to solve for $\gamma$ :

Lemma 29. If an arc-length parameterized crease $\mathbf{x}(s)$ have unique rule segments on one side incident to cone apex $\mathbf{a}$, then an embedding $\mathbf{f}$ is a proper folding if and only if folded curve $\mathbf{X}=\mathbf{f} \circ \mathbf{x}$ is also arc-length parameterized, and rule segments from $\mathbf{a}$ to $\mathbf{x}(s)$ maps isometrically to rule segments from $\mathbf{A}$ to $\mathbf{X}(s)$, where $\mathbf{A}=\mathbf{f} \circ \mathbf{a}$.

Proof. Necessity (only if part) is obvious, so we prove the sufficiency (if part). The folded curve is arc-length parameterized by $s$ as $\frac{d \mathbf{X}(s)}{d s}=\frac{d \mathbf{x}(s)}{d s}=1$, and the length of ruling segment $L(s)$ must be equal $L(s)=\|\mathbf{x}(s)-\mathbf{a}\|=\|\mathbf{X}(s)-\mathbf{A}\|$. Let $\mathbf{r}(s)$ denote the unit ruling vectors from the apex toward the curve in $2 \mathrm{D}$, i.e., $\mathbf{r}(s)=(\mathbf{x}(s)-\mathbf{a}) / L(s)$. Similarly denote unit ruling vector in $3 \mathrm{D}$ by $\mathbf{R}(s)=$ $(\mathbf{X}(s)-\mathbf{A}) / L(s)$. Consider a coordinate system using arc length $s$ and radius $\ell$. The conical portion of the face formed by the crease and a point is uniquely ruled at any point, so $(s, \ell)$ uniquely represent a point on the portion. A point $(s, \ell)$ in $2 \mathrm{D}$ corresponds to $\mathbf{a}+\ell \mathbf{r}(s)$, which is mapped to $3 \mathrm{D}$ to $\mathbf{A}+\ell \mathbf{R}(s)$. Consider a $2 \mathrm{D}$ $C^{1}$ curve $\mathbf{y}(t)$ represented by $(s(t), \ell(t))$, where $t$ is the arc-length parameterization. Then the total derivative of $\mathbf{y}(t)=\mathbf{a}+\ell(t) \mathbf{r}(s(t))$ is

$$
\frac{d \mathbf{y}}{d t}=\frac{\partial \mathbf{y}}{\partial s} \frac{d s}{d t}+\frac{\partial \mathbf{y}}{\partial \ell} \frac{d \ell}{d t}=\ell \frac{d \mathbf{r}}{d s} \frac{d s}{d t}+\mathbf{r} \frac{d \ell}{d t} .
$$

Then, by taking the dot product with itself,

$$
\begin{aligned}
\left\|\frac{d \mathbf{y}}{d t}\right\|^{2} & =\ell^{2}\left\|\frac{d \mathbf{r}}{d s}\right\|^{2}\left(\frac{d s}{d t}\right)^{2}+2 \ell \frac{d \mathbf{r}}{d s} \cdot \mathbf{r}\left(\frac{d s}{d t}\right)\left(\frac{d \ell}{d t}\right)+\|\mathbf{r}\|^{2}\left(\frac{d \ell}{d t}\right)^{2} \\
& =\ell^{2}\left\|\frac{d \mathbf{r}}{d s}\right\|^{2}\left(\frac{d s}{d t}\right)^{2}+\left(\frac{d \ell}{d t}\right)^{2}
\end{aligned}
$$

where we used $\mathbf{r} \cdot \mathbf{r}=1$ and $2 \frac{d \mathbf{r}}{d s} \cdot \mathbf{r}=\frac{d}{d s}(\mathbf{r} \cdot \mathbf{r})=0$. Because $L(s) \mathbf{r}(s)=\mathbf{x}(s)-\mathbf{a}$, taking derivatives yields

By taking the dot product,

$$
L \frac{d \mathbf{r}}{d s}+\frac{d L}{d s} \mathbf{r}=\frac{d \mathbf{x}}{d s}
$$

$$
L^{2}\left\|\frac{d \mathbf{r}}{d s}\right\|^{2}+\left(\frac{d L}{d s}\right)^{2}=\left\|\frac{d \mathbf{x}}{d s}\right\|^{2}=1,
$$

again using $\frac{d \mathbf{r}}{d s} \cdot \mathbf{r}=0$ and $\mathbf{r} \cdot \mathbf{r}=1$. Thus,

$$
\left\|\frac{d \mathbf{y}}{d t}\right\|^{2}=\frac{\ell^{2}}{L^{2}}\left(1-\left(\frac{d L}{d s}\right)^{2}\right)\left(\frac{d s}{d t}\right)^{2}+\left(\frac{d \ell}{d t}\right)^{2} .
$$

The mapped crease $\mathbf{Y}(t)$ in $3 \mathrm{D}$ is defined by $\mathbf{Y}(t)=\mathbf{A}+\ell(t) \mathbf{R}(s(t))$. Then,

$$
\left\|\frac{d \mathbf{Y}}{d t}\right\|^{2}=\frac{\ell^{2}}{L^{2}}\left(1-\left(\frac{d L}{d s}\right)^{2}\right)\left(\frac{d s}{d t}\right)^{2}+\left(\frac{d \ell}{d t}\right)^{2},
$$

similarly using $\mathbf{R} \cdot \mathbf{R}=1, \frac{d \mathbf{R}}{d s} \cdot \mathbf{R}=0$, and $\left\|\frac{d \mathbf{X}}{d s}\right\|^{2}=1$. Therefore $\left\|\frac{d \mathbf{y}}{d t}\right\|^{2}=\left\|\frac{d \mathbf{Y}}{d t}\right\|^{2}=$ 1 and the mapping is isometric.

A similar argument works for cylindrical surfaces. 
Lemma 30. If an arc-length parameterized crease $\mathbf{x}(s)$ have unique rule segments on one side parallel to $\mathbf{r}$, such that $\mathbf{r}$ is perpendicular to segment $c$. then an embedding $\mathbf{f}$ is a proper folding if and only if folded curve $\mathbf{X}=\mathbf{f} \circ \mathbf{x}$ is also arc-length parameterized, and the perpendicular rule segments from $\mathbf{x}(s)$ to $c$ maps isometrically to rule segments from $\mathbf{X}(s)$ perpendicularly to a planar curve $C$, where $C=\mathbf{f} \circ c$.

Proof. Necessity (only if part) is obvious, so we prove the sufficiency (if part). The folded curve is arc-length parameterized by $s$ as $\frac{d \mathbf{X}(s)}{d s}=\frac{d \mathbf{x}(s)}{d s}=1$, and the length of ruling segment $L(s)$ must be equal $L(s)=\|\mathbf{x}(s)-\mathbf{c}(s)\|=\|\mathbf{X}(s)-\mathbf{C}(s)\|$. Let $\mathbf{r}$ denote the unit ruling vectors from the apex toward the curve in $2 \mathrm{D}$, i.e., $\mathbf{x}(s)=$ $\mathbf{c}(s)+L(s) \mathbf{r}$. Similarly denote unit ruling vector in $3 \mathrm{D}$ by $\mathbf{X}(s)=\mathbf{C}(s)+L(s) \mathbf{R}$. Consider a coordinate system using arc length $s$ and length along the ruled segments $\ell$. The face is uniquely ruled between the crease and the curve at any point, so $(s, \ell)$ uniquely represent a point on the portion. A point $(s, \ell)$ in $2 \mathrm{D}$ corresponds to $\mathbf{c}(s)+\ell \mathbf{r}$, which is mapped to $3 \mathrm{D}$ to $\mathbf{C}(s)+\ell \mathbf{R}$. Consider a $2 \mathrm{D} C^{1}$ curve $\mathbf{y}(t)$ represented by $(s(t), \ell(t))$, where $t$ is the arc-length parameterization. Then the total derivative of $\mathbf{y}(t)=\mathbf{c}(s)+\ell(t) \mathbf{r}$ is

$$
\frac{d \mathbf{y}}{d t}=\frac{\partial \mathbf{y}}{\partial s} \frac{d s}{d t}+\frac{\partial \mathbf{y}}{\partial \ell} \frac{d \ell}{d t}=\frac{d \mathbf{c}}{d s} \frac{d s}{d t}+\mathbf{r} \frac{d \ell}{d t} .
$$

Then,

$$
\begin{aligned}
\left\|\frac{d \mathbf{y}}{d t}\right\|^{2} & =\left\|\frac{d \mathbf{c}}{d s}\right\|^{2}\left(\frac{d s}{d t}\right)^{2}+2 \frac{d \mathbf{c}}{d s} \cdot \mathbf{r}\left(\frac{d s}{d t}\right)\left(\frac{d \ell}{d t}\right)+\|\mathbf{r}\|^{2}\left(\frac{d \ell}{d t}\right)^{2} \\
& =\left\|\frac{d \mathbf{c}}{d s}\right\|^{2}\left(\frac{d s}{d t}\right)^{2}+\left(\frac{d \ell}{d t}\right)^{2}
\end{aligned}
$$

where we used $\mathbf{r} \cdot \mathbf{r}=1$ and $\frac{d \mathbf{c}(s)}{d s} \cdot \mathbf{r}=0$. Now differentiate $L(s) \mathbf{r}+\mathbf{c}(s)=\mathbf{x}(s)$ to obtain

By taking the dot product,

$$
\frac{d \mathbf{c}}{d s}+\frac{d L}{d s} \mathbf{r}=\frac{d \mathbf{x}}{d s} .
$$

$$
\left\|\frac{d \mathbf{c}}{d s}\right\|^{2}+\left(\frac{d L}{d s}\right)^{2}=\left\|\frac{d \mathbf{x}}{d s}\right\|^{2}=1,
$$

again using $\frac{d \mathbf{c}}{d s} \cdot \mathbf{r}=0$ and $\mathbf{r} \cdot \mathbf{r}=1$. Thus,

$$
\left\|\frac{d \mathbf{y}}{d t}\right\|^{2}=\left(1-\left(\frac{d L}{d s}\right)^{2}\right)\left(\frac{d s}{d t}\right)^{2}+\left(\frac{d \ell}{d t}\right)^{2} .
$$

The mapped crease $\mathbf{Y}(t)$ in $3 \mathrm{D}$ is defined by $\mathbf{Y}(t)=\mathbf{C}(s)+\ell(t) \mathbf{R}$. Then,

$$
\left\|\frac{d \mathbf{Y}}{d t}\right\|^{2}=\left(1-\left(\frac{d L}{d s}\right)^{2}\right)\left(\frac{d s}{d t}\right)^{2}+\left(\frac{d \ell}{d t}\right)^{2}
$$

similarly using $\mathbf{R} \cdot \mathbf{R}=1, \frac{d \mathbf{C}}{d s} \cdot \mathbf{R}=0$, and $\left\|\frac{d \mathbf{X}}{d s}\right\|^{2}=1$. Therefore $\left\|\frac{d \mathbf{y}}{d t}\right\|^{2}=\left\|\frac{d \mathbf{Y}}{d t}\right\|^{2}=$ 1 and the mapping is isometric.

By Lemmas 29 and 30 , the existence of the folded form is ensured by constructing the folded crease $f(\gamma)$ such that in the folded state, the distance between the $V_{0,1}$ and $f(\gamma(t))$ is always $r(t)$, and the distance from $z x$ plane is always $\ell(t)$. If we view the projection of the curve, this is equivalent to constructing a curve represented 
by polar coordinate $(\theta(t), h(t))(\theta \in \mathbb{R})$, such that (i) the curve has arclength $t$ and (ii) $\theta(t)$ is a monotonic function (in order to avoid self-intersection). Condition (i) yields a differential equation

$$
1=h^{2}\left(\frac{d \theta(t)}{d t}\right)^{2}+h^{\prime}(t)^{2} .
$$

Condition (ii) gives us $0<\frac{d \theta(t)}{d t}$ and $h(t)>0$, so the differential equation becomes

$$
\frac{d \theta(t)}{d t}=\frac{1}{h(t)} \sqrt{1-\left(\frac{d h(t)}{d t}\right)^{2}}
$$

which has solution

$$
\theta(t)=\int_{0}^{t} \frac{1}{h(t)} \sqrt{1-\left(\frac{d h(t)}{d t}\right)^{2}} d t
$$

if and only if $\left(\frac{d h(t)}{d t}\right)^{2} \leq 1$ for $t \in(0,1)$. Combined with condition (ii), $\left(\frac{d h(t)}{d t}\right)^{2}<1$.

$$
\left(\frac{d h(t)}{d t}\right)^{2}=\frac{\left[(t-u)-\frac{1}{2}\left(v-v^{*}\right) \ell^{\prime}(t)\right]^{2}}{(t-u)^{2}+\left(v-v^{*}\right)\left[\frac{1}{4}\left(v+v^{*}\right)-\ell(t)\right]}<1
$$

which is equivalent to

$$
-\frac{1}{4}\left(v-v^{*}\right)\left[1+\left(\frac{d \ell(t)}{d t}\right)^{2}\right]+\left[\frac{1}{2} v-\left(\ell(t)+\frac{d \ell(t)}{d t}(u-t)\right)\right]>0 .
$$

Because $\ell(t)+\frac{d \ell(t)}{d t}(u-t)$ represents the $y$ coordinate of the intersection between the tangent line to $\gamma_{0,0}^{+}$at $t$ and a vertical line passing through $V_{0,1}, \frac{v}{2}-$ $\left(\ell(t)+\frac{d \ell(t)}{d t}(u-t)\right)$ is always positive. Also $1+\left(\frac{d \ell(t)}{d t}\right)^{2}$ is positive, so the condition is given by

$$
v-v^{*}<\frac{4\left[\frac{v}{2}-\left(\ell(t)+\frac{d \ell(t)}{d t}(u-t)\right)\right]}{1+\left(\frac{d \ell(t)}{d t}\right)^{2}} .
$$

If we define $v_{l i m}^{*}<v$ as

$$
v-v_{\text {lim }}^{*}=4\left[\frac{v}{2}-\left(\ell(t)+\frac{d \ell(t)}{d t}(u-t)\right)\right] /\left[1+\left(\frac{d \ell(t)}{d t}\right)^{2}\right],
$$

then there exists a continuous solution for $v^{*}$ in $\left(v_{\text {lim }}^{*}, v\right)$.

Now that we have folded an individual gadget, we can tile the gadget to get a proper folding of the overall crease pattern. Here we use the fact that the oriented folded module, for a sufficiently small fold angle, projects to a kite in the $x y$ plane.

Consider inversions of the oriented folded module through the midpoints of its boundary edges, followed by negating all normals to swap the top and bottom sides of the paper (Figure 10). If we consider the $x y$ projection, the operation corresponds to $180^{\circ}$ rotation around the midpoint of the kite, resulting in a tessellation. Thus, in particular, there are no collisions between the copies of the folded module. Because each connecting edge is mapped onto itself in $3 \mathrm{D}$, this tessellation has no gap in 3D. Also, because the boundary is on a ruled segment, the surface normal vector is constant along each edge. The surface normal is flipped by the inversion, and then negated back to its original vector, so the surface normals at corresponding points 

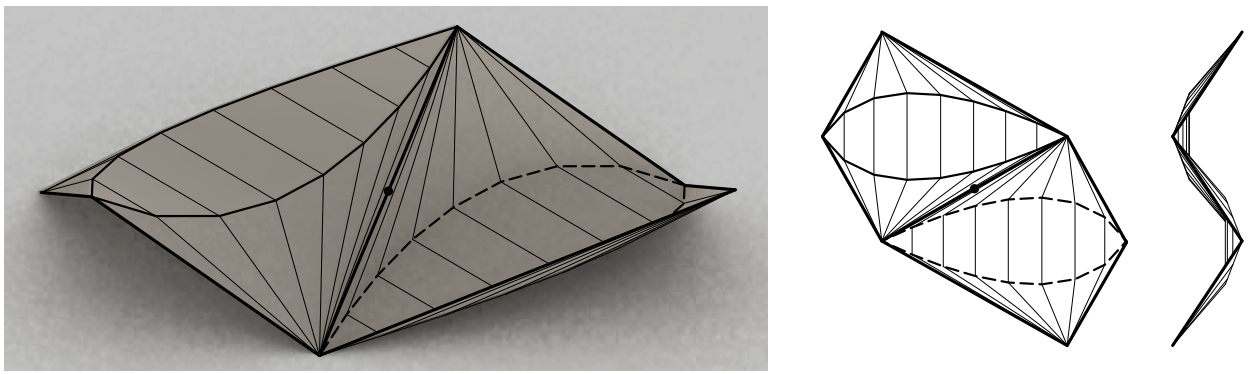

Figure 10. The connection of kite structures.

match. Thus the shared boundaries remain uncreased in the tessellated folding. To show that this tessellated folding comes from one sheet of paper, we can apply the same tiling transformation to the crease-pattern module, which is also a kite, so it tiles the plane with the same topology and intrinsic geometry. Therefore the plane can fold into the infinitely tiled folding.

\section{ACKNOWLEDGMENTS}

We thank the Huffman family for access to the third author's work, and permission to continue in his name.

\section{REFERENCES}

[Demaine and O'Rourke 07] Erik D. Demaine and Joseph O'Rourke. Geometric Folding Algorithms: Linkages, Origami, Polyhedra. Cambridge University Press, 2007.

[Demaine et al. 11] Erik D. Demaine, Martin L. Demaine, Vi Hart, Gregory N. Price, and Tomohiro Tachi. "(Non)existence of Pleated Folds: How Paper Folds Between Creases." Graphs and Combinatorics 27:3 (2011), 377-397.

[Fuchs and Tabachnikov 99] Dmitry Fuchs and Serge Tabachnikov. "More on Paperfolding." The American Mathematical Monthly 106:1 (1999), 27-35. Available online (http://www.jstor. org/pss/2589583).

[Fuchs and Tabachnikov 07] Dmitry Fuchs and Serge Tabachnikov. "Developable Surfaces." In Mathematical Omnibus: Thirty Lectures on Classic Mathematics, Chapter 4. American Mathematical Society, 2007.

[Huffman 76] David A. Huffman. "Curvature and Creases: A Primer on Paper." IEEE Transactions on Computers C-25:10 (1976), 1010-1019.

(E. Demaine and M. Demaine) Computer Science and Artificial Intelligence Laboratory, Massachusetts Institute of Technology, 32 Vassar St., Cambridge, MA 02139, USA

E-mail address: demaine@mit.edu

(D. Huffman) Department of Computer Science, University of California, Santa Cruz, CA 95064, USA.

(D. Koschitz) School of Architecture, Pratt Institute, 200 Willoughby Ave., BrookLYN, NY 11205, USA.

E-mail address: duks@pratt.edu

(T. Tachi) Department of General Systems Studies, The University of Tokyo, 3-8-1 Komaba, Meguro-Ku, Tokyo 153-8902, Japan

E-mail address: tachi@idea.c.u-tokyo.ac.jp 